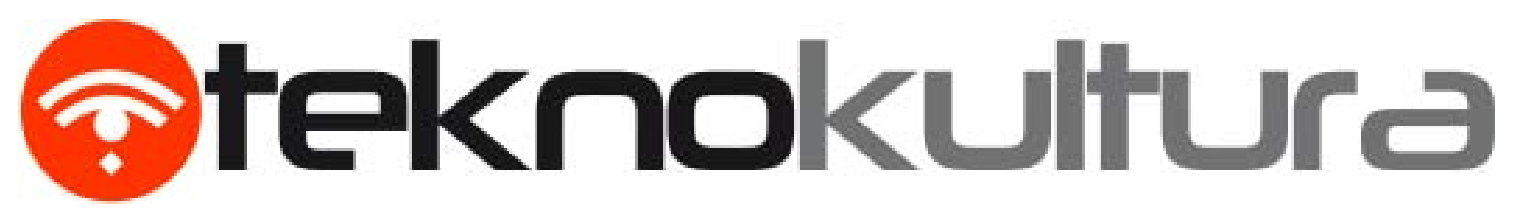

\#Tabarés GutiérRez. R. (2016). El surgimiento de HTML5; un nuevo paradigma en los estándares Web, Revista Teknokultura Vol. 13(1), 169-192.

Recibido: 23-03-2016

Aceptado: 25-04-2016
Open peer review

http://revistas.ucm.es/index.php/TEKN/pages/view/opr-52152

\title{
El surgimiento de HTML5; un nuevo paradigma en los estándares Web
}

\section{The rise of HTML5; a new paradigm for Web standards}

\author{
Raúl Tabarés Gutiérrez \\ TECNALIA \\ faraondemetalegmail.com
}

\section{Resumen}

Gracias al crecimiento, expansión y popularización de la World Wide Web, su desarrollo tecnológico tiene una creciente importancia en la sociedad. La simbiosis que protagonizan estos dos entornos ha propiciado una mayor influencia social en las innovaciones de la plataforma y un enfoque mucho más práctico. Nuestro objetivo en este artículo es describir, caracterizar y analizar el surgimiento y difusión del nuevo estándar de hipertexto que rige la Web; HTML5. Al mismo tiempo exploramos este proceso a la luz de varias teorías que aúnan tecnología y sociedad. Dedicamos especial atención a los usuarios de la World Wide Web y al uso genérico que realizan de los Medios Sociales o "Social Media". Sugerimos que 
el desarrollo de los estándares web está influenciado por el uso cotidiano de este nuevo tipo de tecnologías y aplicaciones.

\title{
Palabras Clave
}

SCOT; innovación de usuario; social media; WhatWG; innovación social.

\begin{abstract}
Due to the growth, expansion and popularization of the World Wide Web, its technological development has a growing importance in society. The symbiosis that generates both of these elements has delivered a greater social influence to the innovation processes and a much more practical approach. In this paper we would like to describe, to characterize and to analyze the growth and diffusion of the new web hypertext standard called HTML5. Moreover, we explore this process in light of various theories about technology and society. Special attention is paid to World Wide Web users and Social Media. We claim that web standards are influenced through the daily use of these kinds of new technologies.
\end{abstract}

\section{KEYWORDS}

SCOT; user-driven innovation; social media; WhatWG; social innovation. 


\section{Contenidos}

1. Introducción

2. El desarrollo de HTML5

3. La creación de HTML5

4. Las mejoras de HTML5

5. La creación de HTML5 desde un punto de vista social

6. Difusión e innovación social en HTML5

7. ¿Construcción social o tecnología autónoma?

8. El papel de los usuarios

9. Conclusiones

10. Referencias

\section{Contents}

1. Introduction

2. The development of HTML5

3. The origin of HTML5

4. HTML5 assets

5. HTML5 from a social approach

6. Social innovation and social diffusion of HTML5

7. Social construction or autonomous technology?

8. The role of the users

9. Conclusions

10. References 



\section{Introducción}

La creación y desarrollo de estándares web afronta un punto de inflexión debido a la mayor presencia e influencia de los dispositivos móviles y los medios sociales en la sociedad. Si bien es cierto que la World Wide Web se basa en tres tecnologías: el protocolo HTTP (Hypertext Transfer Protocol), el localizador URI (Unified Reference Indentificator) y el lenguaje de marcado HTML (Hypertext Markup Language). Constatamos que solamente esta última ha afrontado recientemente grandes cambios en su desarrollo tecnológico debido al número de interrelaciones sociales observadas en su conceptualización y progreso. Por ello, creemos que su estudio desde un punto de vista que aúne tecnología y sociedad puede ser de sumo interés. Este artículo describe el origen y desarrollo de HTML5, enuncia sus características más disruptivas y analiza sus principales hitos. Nuestro ánimo en este artículo es contrastar teorías que explican la dinámica social de la tecnología (como el modelo SCOT) con otras que enuncian su pérdida de control por parte de la sociedad (como la Tecnología Autónoma), y al mismo tiempo evidenciar el papel cada vez más importante que juegan los usuarios en el desarrollo tecnológico. Sugerimos que el usuario medio ha ejercido una gran influencia en el desarrollo de estándares de hipertexto.

\section{EI desarrollo de HTML5}

\subsection{La creación de HTML5}

El nacimiento de HTML5 constituye un punto de inflexión en el modo que se desarrollan los estándares web. Su origen radica en un pequeño grupo de profesionales disconformes con el rumbo que había decidido tomar la organización que vela por el desarrollo de los estándares; el W3C (Word Wide Consortium). Un grupo de desarrolladores de la Fundación Mozilla y de Opera Software, a los que se les unen más tarde profesionales de Apple, fundan en 2004 el Web Hypertext Application Technology Working Group (WhatWG) al margen del W3C (Franganillo, 2010).

Este hecho sucede en junio de 2004 cuando el W3C alberga un seminario sobre "Web Applications and Compound Documents" ${ }^{1}$. En esta conferencia se dieron cita distribuidores

\footnotetext{
${ }^{1}$ Se puede consultar los detalles de este workshop en: <http://www.w3.org/2004/04/webapps-cdf-ws/> (Accedido por última vez el 23/12/2013).
} 
de navegadores web, compañías de desarrollo web y otro tipo de agentes que integraban el $\mathrm{W} 3 \mathrm{C}$ en esos momentos. Durante este evento, profesionales de Opera y Mozilla realizaron una presentación sobre su visión del futuro de la Web. Esta se podía resumir en una premisa; evolucionar el HTML4 existente en un estándar capaz de incluir nuevas características para los desarrolladores de aplicaciones web (Pilgrim, 2010). Además, subrayaron la importancia de su propuesta en torno a 7 principios (The Mozzilla Foundation \& Opera Software, 2004);

- Backwards Compatibility, clear migration path: Las tecnologías web que se utilicen deben ser familiares para los desarrolladores (HTML, CSS, DOM y JavaScript). Las características a implementar y las nuevas funcionalidades a soportar deben ser en base a las que soporta el navegador Internet Explorer 6, ya que es el que mayor cuota de mercado tiene. Y por lo tanto, a lo que la mayoría de los usuarios están acostumbrados.

- Well-defined error handling: El manejo de los errores en las aplicaciones web debe ser definido a un detalle en el cual los desarrolladores no tengan que inventárselos o utilicen procesos de reingeniería basándose en cómo los maneja la competencia.

- Users should not be exposed to authoring errors: Las especificaciones deben especificar exactamente cómo se deben recuperar los errores para cada escenario posible.

- Practical use: Cada nueva aplicación web que se genere debe ser justificada por un uso práctico (Sin que tenga la misma validez en el caso contrario).

- $\quad$ Scripting is here to stay: Pero también debe evitarse cuando pueda ser sustituido por el "lenguaje de marcas".

- Device-specific profiling should be avoided: Los desarrolladores deben ser capaces de implementar las mismas funcionalidades de la página, ya sea accedida a través de un PC o a través de un dispositivo móvil.

- Open process: La Web se ha beneficiado durante su desarrollo de un entorno abierto. Las aplicaciones web serán de suma importancia y por lo tanto, su desarrollo deberá ser realizado de una manera abierta (listas de correo, drafts, etc).

También se sondeó la opinión de los asistentes mediante una encuesta. Ian Hickson (Opera Software) preguntó expresamente: “¿Debería el W3C desarrollar extensiones aclarativas para HTML y CSS e imperativas para DOM, con el fin de hacer frente a los requisitos medios de las aplicaciones web, y en contra de los sofisticados requisitos de las API's nativas de cada sistema operativo?” (The Mozzilla Foundation \& Opera Software, 2004)

Esta proposición se sometió a votación pero fue rechazada por 11 votos en contra y 8 a favor. Además de esta negativa, el W3C hizo una declaración formal en la que indicaba que 
no iba a prestar apoyo, ni ofrecer recursos a ninguna propuesta que no estuviera dentro de los grupos de trabajo que tenía establecidos en esa época (Pilgrim, 2010). Por ello, este grupo de personas decidió continuar su camino fuera del W3C. Registraron el dominio whatwg.org y comenzaron a trabajar en su propuesta fundando el What $W G^{2}$. Este grupo de trabajo, abierto a la colaboración de terceros, estaba formado por fabricantes de navegadores y agentes interesados en volver a los orígenes de HTML, sin ahondar en lenguajes nuevos como XHTML. Su enfoque se basaba en garantizar la compatibilidad regresiva o retro-compatibilidad ${ }^{3}$ del nuevo estándar, lo cual es muy apreciado entre los usuarios y desarrolladores de lenguajes de programación.

Sin embargo, el W3C quería proseguir con el desarrollo de XHTML a pesar de que no disponía de compatibilidad regresiva con HTML. Necesitaba de un MIME type nuevo ${ }^{4}$ lo cual suponía desechar el trabajo realizado anteriormente con HTML. Tradicionalmente los navegadores siempre se "han olvidado" de los errores sintácticos de HTML. Hasta entonces nadie se había preocupado por su especificación. La historia del desarrollo web hasta este momento se había basado en buena parte en cómo los diferentes desarrolladores de navegadores hacían compatibles sus productos con los de los competidores ignorando las especificaciones y estándares. La mayoría de los navegadores se centraban en presentar de la mejor manera posible "las sopas de etiquetas" que se creaban (Andersson, 2007). El WHAT Working Group dedicó 5 años de trabajo documentando adecuadamente cómo analizar y diseccionar HTML, de un modo compatible con las páginas existentes ${ }^{5}$ (Pilgrim, 2010). Aparte de esta ingente tarea, este grupo también trabajó en el soporte nativo de audio $\mathrm{y}$ video (sin plugins adicionales), en la nueva etiqueta canvas (que permite dibujar directamente sobre la estructura del documento) y en otra especificación en torno a aplicaciones web.

E1 WhatWG consideraba que el enfoque del W3C era demasiado académico. Por ello, creían necesario una nueva versión del estándar desde un punto de vista eminentemente práctico. El W3C sin embargo, parecía estar buscando en diferentes tecnologías un sustituto a HTML (especialmente en XHTML 2.0) (O'Mara, 2012).

\footnotetext{
${ }^{2}$ Se puede consultar la web que alojó este grupo de trabajo alternativo en: $<$ http://www.whatwg.org/news/start> [Consultado el 17/03/2016].

3 Traducido del inglés: Backwards Compatibility.

${ }^{4}$ Los MIME Types (Multipurpose Internet Mail Extensions) constituyen la especificación del tipo de documento que se intercambia. Se señala en el comienzo del código de las páginas web.

${ }^{5}$ Este documento de referencia sigue actualizándose y puede consultarse en: $<$ http://www.whatwg.org/specs/web-apps/current-work/multipage/parsing.html > [Consultado el 17/03/2016].
} 
Dos años y medios después de esa conferencia, XHTML2 languidecía, mientras las nuevas características de HTML levantaban grandes expectativas. A este hecho se unían las voces críticas que acusaban al $\mathrm{W} 3 \mathrm{C}$ de un avance lento y pocos resultados concretos (Castro, 2007). Por ello, en octubre de 2006, Tim Berners-Lee y el W3C anunciaron que trabajarían junto al WhatWG para añadir nuevas funcionalidades y evolucionar de manera conjunta HTML (Berners-Lee, 2006).

En octubre del 2009 el W3C cerraría el grupo de trabajo en torno a XHTML2 (Le Hegaret, 2009) y pararía completamente sus actividades para volcarse de lleno en el desarrollo de HTML5. El primer working draft vería la luz en 2008 (Hickson \& Hyatt, 2008) gracias a la unión de esfuerzos de las respectivas organizaciones. Posteriormente, los navegadores comienzan a soportar HTML5 (Mozilla Firefox es el primero) dando paso a una fase de difusión y concienciación en torno a esta tecnología. Desde entonces, el desarrollo de HTML5 ha proseguido de manera imparable convirtiéndose primero en una " $W 3 C$ Candidate Recommendation" (W3C, 2011) y oficializándose posteriormente el 28 de octubre de 2014 (W3C, 2014).

\subsection{Las mejoras de HTML5}

Lo primero que debemos especificar en torno a HTML5 es que no es una tecnología, sino un conjunto de tecnologías. Hasta ahora, junto al protocolo HTTP y al identificador URI, HTML formaba un trío de tecnologías clave para la World Wide Web. El nuevo paradigma engloba una serie de tecnologías que se recogen bajo un mismo estándar pero que multiplican en gran medida sus posibilidades. Se han introducido multitud de elementos dinámicos, en forma de "etiquetas", que reconfiguran el entorno web y sus contenidos. La especificación de HTML5 no se compone de una sintaxis únicamente, sino que admite dos: HTML y XHTML (Extensible HyperText Markup Language). De este modo los desarrolladores pueden elegir entre un enfoque práctico y poco riguroso (HTML) o un enfoque académico y estricto (XHTML). Esto es debido a que el W3C ha aceptado que HTML y XHTML sean recomendaciones paralelas y puedan coexistir (Franganillo, 2010). HTML5 no está basado en SGML (Standard Generalized Markup Language) y está diseñado para que tenga compatibilidad regresiva tanto con HTML4 como con XHTML1 (Chau, 2009).

Además de estructurar los documentos, HTML5 también especifica cómo se deben interpretar los errores. Esto supone el fin de la "guerra entre navegadores" en torno a la subsanación de los errores (Andersson, 2007; Keith, 2010). Asimismo, incorpora nuevos elementos destinados a ordenar y enriquecer la presentación de los documentos a través de 
elementos de carácter semántico (por ejemplo, article, header, hrgroup, nav, section, aside y footer). Los blogs han influido en gran medida en la conceptualización de estos nuevos elementos (Schafer, 2010), confiriendo dinamismo en la estructura y contenidos web además de la necesidad de establecer una cronología para poder organizar la información.

Aparte de estos elementos, los aspectos más innovadores de la sintaxis de HTML5 son sus elementos multimedia. Muchos de ellos han sido creados tras agrias polémicas y la liberación de codecs en formato propietario (Linder, 2013; Schonfeld, 2010). Algunas de las etiquetas más reseñables e innovadoras son; audio, video, canvas o geolocation. Además de los elementos dinámicos que introduce HTML5 en su sintaxis también hay otros dos lenguajes que complementan el espectro de este conjunto de innovaciones: CSS3 (Cascading Style Sheets), que es el lenguaje que regula los aspectos de estilo de las páginas web y Javascript, que permite programar acciones dinámicas y facilitar la interacción con el usuario. Por último, también se ha lanzado una nueva versión del DOM (Document Object Model) que consiste en la colección de objetos que representan los elementos del lenguaje HTML en la página web.

\section{La creación de HTML5 desde un punto de vista social}

El proceso que da origen al desarrollo de HTML5 es importante por su idiosincrasia. Muchas veces se ha incidido en el hecho de que la industria de los navegadores no ha respetado los procesos de estandarización. De hecho, han promovido innovaciones que han generado muchos problemas de cara a la normalización de los lenguajes implicados (Gutiérrez, 2012). No obstante la negativa del W3C por apostar por una nueva versión de HTML propicia el grupo disidente que lideran profesionales de compañías como Opera, Mozilla y Apple. Estos profesionales lideran la iniciativa WhatWG para desarrollar una nueva versión de HTML que incorpore elementos dinámicos. En especial API's ${ }^{6}$, las cuales son vitales para crear las nuevas aplicaciones web que habían sido popularizadas al albor del fenómeno conocido como "Web 2.0".

El objetivo de este apartado es analizar el proceso anteriormente descrito desde un punto de vista social. Así, se han establecido tres epígrafes en los cuales se abordan diversos enfoques relacionados con la sistemática del proceso innovador.

6 API, del inglés Application Programation Interface, es una Interfaz de Programación de Aplicaciones que permite interactuar a un software con otro, gracias a un conjunto de funciones y procedimientos que se ofrecen a través de una biblioteca de recursos. 


\subsection{Difusión e innovación social en HTML5}

La difusión social de las innovaciones es un elemento clave en su popularización y adopción social. Uno de los primeros autores en investigar el fenómeno de la difusión social de la innovación fue el sociólogo rural británico Everett Rogers. Este autor publicó en 1962 el libro titulado Diffusion of Innovations. En esta obra se definía el proceso de difusión social como: "el proceso mediante el cual una innovación es comunicada a miembros de un sistema social a través de ciertos canales a lo largo del tiempo" (Rogers, 1962, p.5).

Figura 1: Logotipos de HTML5, JaVASCRIPT y CSS3

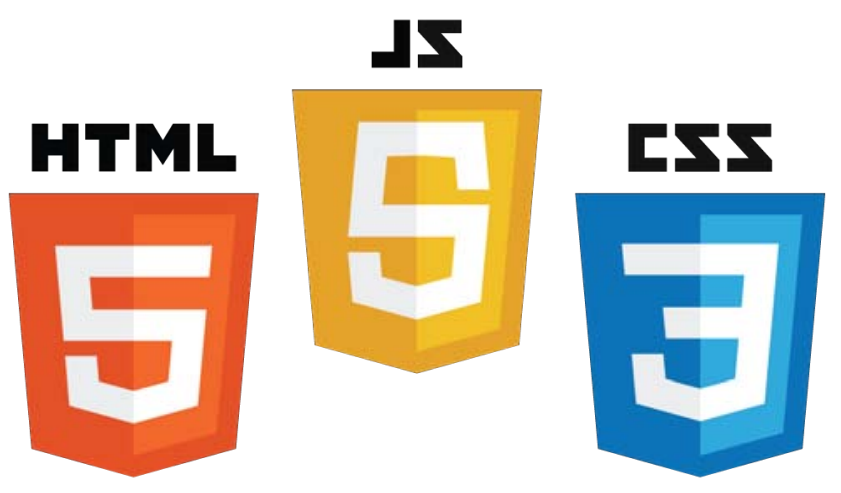

Fuente: W3C

El caso de HTML5 no ha sido ajeno a esta lógica si atendemos a los múltiples esfuerzos realizados para divulgar las ventajas de este nuevo paradigma innovador. La difusión social de HTML5 ha sido un proceso en el cual han estado involucrados tanto instituciones como el W3C, compañías privadas como Apple y Google, fundaciones sin ánimo de lucro, como Mozilla, y personalidades de la talla de Tim Berners-Lee (Berners-Lee, 2006). Todos ellos han obrado para que el cambio de paradigma innovador que suponía HTML5 fuera mayoritariamente adoptado. Si esta innovación no hubiese sido aceptada y adoptada es de suponer que no hubiera tenido éxito (Echeverría, 2013), ya que una innovación sin difusión no tiene ningún impacto económico (OECD, 2005) y por lo tanto, no se populariza. Asimismo, una percepción pública negativa también puede ser una barrera infranqueable para esa innovación (López Cerezo \& González, 2013). Por ello, una vez que el W3C accedió a colaborar en el desarrollo de HTML5 se realizaron varios esfuerzos en materia de difusión de la inno- 
vación. Prueba de ello son las campañas de marketing desarrolladas por el W3C, enfocadas en la difusión del estándar, y que han contado con el desarrollo de logos específicos de HTML5, CSS3 y JavaScript (Figura 1). A su vez, otras figuras públicas como Steve Jobs apoyaron públicamente el nuevo estándar (Jobs, 2010) y fueron más allá cuando denegó el soporte a Flash en los dispositivos móviles de su compañía con el fin de promover el desarrollo de soluciones basadas en este estándar (Tabarés-Gutiérrez, 2015).

El proceso de creación de HTML5 también constituye una innovación social cuando cuestiona el statu quo de los estándares. Además de cuestionar al W3C como órgano de referencia, promueve un proceso participativo para apoyar el desarrollo de una nueva versión de HTML de acuerdo a unos principios abiertos y comunes. El proceso de creación de HTML5 no se limita a un proceso de desarrollo tecnológico al uso, sino que hay una compilación de saberes explícitos e implícitos que se mezclan con las innovaciones técnicas (Innerarity \& Gurrutxaga, 2009) que se desarrollan en este periodo de tiempo. La creación de HTML5 responde a la redefinición de un problema existente y no por la identificación de un problema nuevo. La diferencia es que esta redefinición se hace desde un marco conceptual apropiado. Por ello, creemos que la creación de HTML5 abre la puerta a un nuevo paradigma de la innovación en estándares, al generarse desde abajo hacia arriba, y volviendo a los propios valores que gestaron la Web. HTML5 asienta a su vez las bases para crear nuevas innovaciones en torno a unos principios sociales y tecnológicos, que deben ser consensuados y respetados, para garantizar la cohesión y el desarrollo tecnológico sostenible.

Por último, el surgimiento de HTML5 supone también un desarrollo de software libre al igual que los anteriores estándares de hipertexto. Software que se basa en el libre uso y disponibilidad universal de los contenidos, proponiendo la total libertad de su utilización, modificación, reutilización y redistribución del código fuente del software (Alonso \& García, 2012). En la Web se utilizan diversos desarrollos de software libre como los gestores de contenidos Wordpress o Joomla, navegadores como Mozilla Firefox, bases de datos como MYSQL o servidores web como Apache. Estas aplicaciones han sido utilizadas en la creación de proyectos de un marcado carácter de producción social (Benkler, 2006) como Wikipedia o Linux. Iniciativas que son constitutivas de lo que se conoce como "procomún digital" y que se caracterizan por un régimen de propiedad que no se inscribe ni en lo público, ni en lo privado. Como muy bien apunta Antonio Lafuente (2007); "los nuevos procomunes se hacen visibles cuando esos bienes que son de todos y no son de nadie comienzan a estar amenazados; en torno a ellos surge entonces una comunidad de personas concernidas por el sostenimiento de esos recursos" (Estalella, Rocha, \& Lafuente, 2013, 
p.26). Creemos que el caso que nos ocupa es paradigmático y constituye también un nuevo procomún digital.

\section{2. ¿Construcción social o tecnología autónoma?}

Al revisar el proceso de conceptualización y desarrollo de HTML5 llama poderosamente la atención la cantidad de actores implicados en su desarrollo, el número de lenguajes de programación, marcado y diseño (itinerarios tecnológicos a tomar) y las diversas interacciones que se producen entre estos agentes y las posibles trayectorias tecnológicas. Por ello, a la hora de recoger los fragmentos que la historia tecnológica de este desarrollo ha dejado por el camino no es fácil elegir una metodología o un marco de actuación de cara a realizar un análisis lo más preciso posible. Este es un tema que se ha repetido a lo largo de la historia de los Estudios Sociales de Ciencia y Tecnología y para el que ha habido varias escuelas y corrientes que han intentado dar su propia explicación de la dinámica del cambio tecnológico (Hackett, Amsterdamska, Lynch, \& Wajcman, 2007). Durante décadas los estudios del desarrollo tecnológico han interpretado la tecnología en un sentido mucho más amplio que el que puede referirse a un artefacto o una herramienta en particular. Esta búsqueda por encontrar una definición lo más completa posible ha generado una gran cantidad de literatura al respecto y ha expandido nuestra percepción utilitarista y artefactual de la tecnología (González García, López Cerezo, \& Luján, 1996). Entre otros autores que han promovido una comprensión más holística de lo que significa el concepto de técnica y tecnología nos gustaría mencionar a Jacques Ellul (Ellul, 1964) y a Miguel Ángel Quintanilla (Quintanilla, 1989). Estos dos autores han introducido en sus dos definiciones de técnica el concepto de eficiencia, el cual se convierte en uno de los resortes de la dinámica social. Los autores anteriormente mencionados sirven de introducción al modelo SCOT (Social Construction of Technology). Este modelo contempla a los usuarios de la tecnología como un grupo social y que juega un papel fundamental en el desarrollo de las tecnologías involucradas. Este modelo desarrollado por Bijker, Hughes, \& Pinch (1987) supuso un punto de inflexión en los estudios $\mathrm{CTS}^{7}$ a la hora de explicar la dinámica y caracterización del desarrollo tecnológico. En este enfoque la idea de "sistema tecnológico" juega un papel muy importante a la hora de comprender la dimensión social de la tecnología (Hughes, 1987). Ese "sistema tecnológico" es bastante similar a lo que Quintanilla denomina como "sistema técnico" (Quintanilla, 1998).

\footnotetext{
${ }^{7}$ Ciencia, Tecnología y Sociedad.
} 
Por otro lado, Bijker también ahonda en el concepto de tecnología y establece tres capas diferentes de significado: artefactos físicos, actividades humanas y conocimiento (Bijker, 1995). En estas concepciones de técnica, tecnología, sistema técnico y sistema tecnológico residen no sólo artefactos, sino también sujetos sociales, sistemas sociales y diversos tipos de conocimientos. Queremos hacer hincapié en este "sentido social" que tiene el desarrollo de la tecnología, ya que el caso que nos ocupa del desarrollo del nuevo estándar HTML5 creemos que encaja perfectamente bajo este marco teórico.

El desarrollo del estándar no se lleva a cabo por un modelo de desarrollo tecnológico o de "innovación lineal" (Lundvall, 1992). Es fruto de un proceso iterativo que permite una retroalimentación de diversos actores y paradigmas tecnológicos conformando un proceso de producción social (Benkler, 2006). El W3C es la organización que se encarga del desarrollo de estándares para la Web pero debido al estancamiento de sus propuestas en torno a XHTML y su difícil encaje fueron debilitándose en favor de la propuesta del WhatWG. En el modelo SCOT se enfatiza que la tecnología no determina el curso de la acción humana, sino más bien al contrario. La forma final de un artefacto tecnológico es el resultado de un proceso social en el que diversos grupos sociales juegan un papel crucial en la selección de las variantes a elegir (Bijker \& Pinch, 1984) posibilitando la supervivencia de unas trayectorias y la extinción de otras. Este proceso es al que Bijker y Pinch enuncian como la "flexibilidad interpretativa" de la tecnología (Bijker et al., 1987; Bijker \& Pinch, 1984). Diferentes grupos de usuarios pueden construir diferentes significados para con la tecnología, pero a medida que el paradigma dominante se abre paso, los demás significados se desvanecen. Llegar a entender el papel que juegan estos grupos de usuarios líderes (Von Hippel, 1998) es clave. Otros autores también han considerado a los usuarios como un actor relevante en el desarrollo de la tecnología (Latour, 1992) y han ejercido una gran influencia en el modelo SCOT (Williams \& Edge, 1996).

En estos y otros modelos destaca la influencia de factores sociales en el desarrollo exitoso de la tecnología. En el caso que nos ocupa, los expertos que conforman el WhatWG y sus relaciones personales constituyen un factor decisivo en el establecimiento del paradigma de HTML5 y de los valores que subyacen en él. Este último punto nos lleva a tener que hablar de la relación de las tecnologías y los valores. Un tema que toma forma expresa en un famoso artículo de Langdon Winner titulado; "Do artifacts have politics?" (Winner, 1980). Este autor realizaría posteriormente una influente crítica del modelo SCOT, por lo descriptivo y analítico del mismo, de cara al desarrollo tecnológico (Winner, 1993). Pese a que el modelo SCOT es de gran ayuda para describir la dinámica del desarrollo tecnológico --como el propio Winner admite--, hay que aludir también al concepto de "tecnología autónoma" (Winner, 1979). Y es que la historia de HTML5 es una reacción en cierto modo a la 
situación anómala que se produce en el desarrollo de estándares debido al fenómeno "Web $2.0 "$.

La idea de tecnología autónoma "sirve de etiqueta a todas las concepciones y observaciones en el sentido de que la tecnología escapa de algún modo al control humano" (Winner, 1979, p.25). Como argumenta el filósofo norteamericano, ciertas técnicas son capaces de desencadenar efectos no previstos o deseados. Esto es a lo que Winner se refiere como una peculiaridad de la tecnología del siglo XX; "la pérdida del dominio (sobre la tecnología) se manifiesta en una mengua en nuestra habilidad para conocer, juzgar o controlar nuestros medios técnicos" (Winner, 1979, p.38).

Lo cierto es que la corta pero intensa historia de la Web está llena de episodios en los que el desarrollo de estándares es siempre uno de los mayores perjudicados. Esta pérdida del dominio tecnológico viene representada por varios factores que se dan en el desarrollo de la Web, por motivos muy diversos, intereses encontrados y agentes dispares. Ejemplo de esta pérdida del dominio tecnológico es la proliferación de software propietario como Flash o Silverlight a lo largo del periodo conocido como "Web 2.0". Esta expansión constituyó una gran amenaza a la interoperabilidad de la plataforma que en su día ideó Tim BernersLee. Otros episodios anteriores a este periodo de popularización de la Web como la famosa "Guerra de Navegadores" (Gutiérrez, 2012) también nos han dejado innovaciones cuando menos extrañas. Es el caso de la etiqueta $M A R Q U E E^{8}$. Esta clase de mejoras son una de las principales armas que utilizan los distribuidores de software y navegadores web para presionar e ir más allá de los estándares. La justificación de este énfasis por la mejora continua de las aplicaciones es debido a que si dichas nuevas funcionalidades son aceptadas y valoradas por una mayoría de usuarios de la Web suelen traducirse en una mayor cuota de mercado. Por ello, la mayoría de compañías que basan su modelo de negocio en la Web presionan en esta dirección. El papel del W3C siempre ha sido velar por la naturaleza de la Web e Internet, controlando la incorporación de elementos externos o extraños al estándar.

\footnotetext{
${ }^{8}$ Las etiquetas Marquee (IE) y Blink (Netscape) son dos ejemplos de cómo a veces el empuje de las compañías por innovar y ofrecer algo diferente al usuario puede convertirse en algo problemático que eche por tierra el trabajo ya realizado. Estas etiquetas se caracterizan por ofrecer textos que continuamente se mueven en la pantalla. Lo cual suponía un auténtico quebradero de cabeza para los usuarios que querían consultar información, sin cons tantemente tener distracciones en la pantalla. Además, también presentaban bastantes problemas en la optimización del código. Se puede consultar en la página de la Wikipedia en inglés esta información. $<$ http://en.wikipedia.org/wiki/Marquee_element> [Consultado el 25/09/2012]
} 


\subsection{El papel del usuario medio}

Por último, en esta visión social del fenómeno HTML5 nos gustaría prestar atención al usuario medio o no técnico de la Web. Hemos constatado como a través del WhatWG se promueve un cambio a favor de la re-estandarización de la Web y el abandono de elementos externos o de software propietario. No obstante creemos que una de las mayores fuerzas de cambio que posee la World Wide Web son sus propios usuarios; usuarios que carecen de conocimientos técnicos de desarrollo web, pero que son el público mayoritario de la gran cantidad de plataformas de social media que surgen durante el fenómeno conocido como "Web 2.0". Este fenómeno abarca desde finales de la década de los 90 a finales de los 2000 (Figura 2), una época caracterizada por un gran incremento de usuarios de la Web. La popularización de la plataforma se produce a través de la aparición de aplicaciones colaborativas y que posteriormente se catalogarían como medios sociales. Este hecho supone un punto de inflexión en la historia de la Web, ya que contribuye en gran medida a su apropiación social.

\section{Figura 2: Crecimiento de usuarios de Internet desde 1996}

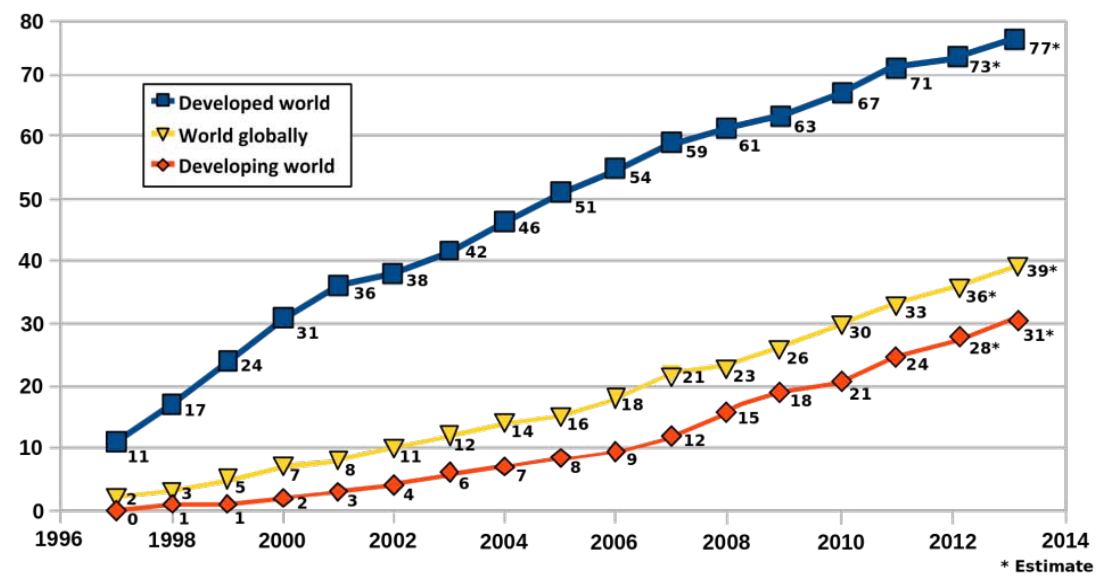

Fuente: Wikipedia.

Tim Berners-Lee, creador de la Web, ha manifestado en varias ocasiones su contrariedad a que la primera versión de la plataforma no tuviera esa centralidad en las personas (Laningham, 2006). En sus primeras etapas la Web es percibida como un medio en el cual solo 
podían publicar contenidos un reducido número de personas ${ }^{9}$ con los conocimientos necesarios (P. Anderson, 2007). Será con la llegada de la Web 2.0 que la propia Web empieza a percibirse como un producto de masas (Duggan \& Smith, 2014).

Figura 3: Horas de vídeo subidas a YouTube desde junio de 2007 a mayo de 2013

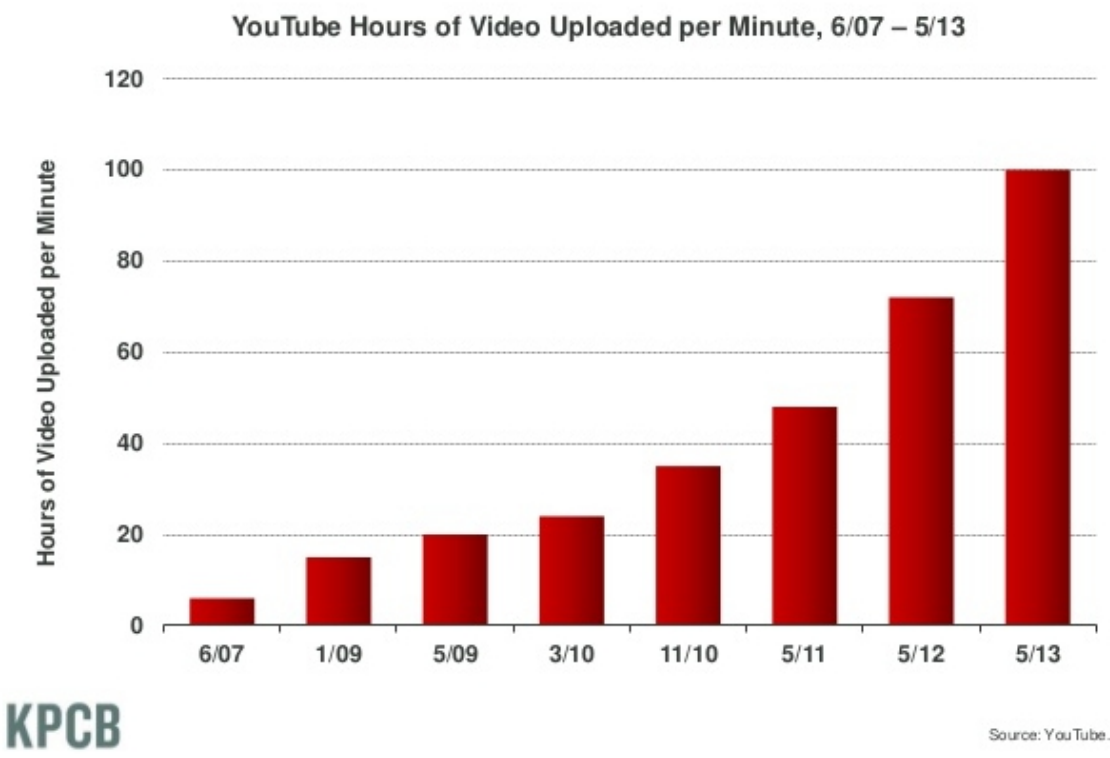

Fuente: $K P C B$ a través de YouTube.

Además de atraer un mayor número de usuarios, la Web 2.0 también produce un gran cambio en el tipo de contenidos que se producen ya que a partir de este momento el multimedia (fotos, videos, etc.) pasa a cobrar un mayor protagonismo (Figura 3). Los usuarios que no disponen de los conocimientos necesarios de edición web (html, css, javascript, php, etc.) ven en plataformas como Facebook, Blogger, YouTube o Flickr, los instrumentos que les permiten crear y compartir información. Las "tecnologías sociales" (Helmer, Brown, \& Gordon, 1966) que aparecen en este periodo constituyen la "caja de herramientas" que necesitaba el usuario no técnico para poder generar contenido e interactuar con otros usuarios.

9 Quizás, el origen del problema pueda estar en el propio desarrollo tecnológico inicial de la Web y en los primeros navegadores que se desarrollan; ViolaWWW, Mosaic y Netscape, los cuales no traen integrada por defecto, una función de editar (Netscape en sus últimas versiones sí que implementaría de forma opcional un editor de HTML). 
Este tipo de procesos capacitadores, de cara a los usuarios, es lo que algunos autores han identificado como una “democratización de la innovación” (Von Hippel, 2005).

En nuestra opinión, estos primeros usuarios de este tipo de aplicaciones o "lead users" (Von Hippel, 1998) son los principales causantes de la popularización de la Web. La generalización de este tipo de servicios, de cara a crear y compartir contenidos, unidos a otros condicionantes como el "efecto red" (Klemperer, 2006; Liebowitz \& Margolis, 1994; Shapiro \& Varian, 1999), "la economía de la larga cola" (C. Anderson, 2004, 2007), el fomento de una "arquitectura de participación" (O’Reilly, 2005) e incluso la consolidación en la Web de "tecnologías fáticas" (Tucker, Wang, \& Haines, 2012; Wang, Tucker, \& Rihll, 2011) producen el ecosistema idóneo para que los usuarios no técnicos ${ }^{10}$ puedan crear y compartir fácilmente información en la World Wide Web. A partir de la eclosión del fenómeno conocido como "Web 2.0" se rebajan las barreras de entrada de uso de las aplicaciones. Se promueve la sencillez de su uso en entornos más ligeros (que no consumen mucho ancho de banda) y que demuestren su interoperabilidad a través de API's. Muchas veces explotando datos de otros servicios con el objetivo de que terceros puedan desarrollar "mash-up's""11 (P. Anderson, 2007).

Este nuevo ecosistema hace posible que los usuarios también puedan editar la Web (aunque no directamente) y de este modo empezar a innovar en ella. El "Social Media" o "Medios Sociales" se convierte en la herramienta del usuario medio para poder crear y compartir contenidos. Pero además, también es el elemento con el cuál se influye en el desarrollo tecnológico. Esto se debe a la mayor predominancia de elementos multimedia, lo cual hace necesario un mayor conjunto de elementos nativos de estandarización. Y es que si se abusa de software propietario se ralentiza la experiencia de usuario y la interoperabilidad entre servicios. También podemos decir que, al fin y al cabo, son los clásicos criterios de eficiencia de un sistema técnico los que residen en esta argumentación (Ellul, 1964; Quintanilla, 1989). Otros autores han definido este tipo de influencia también como "cultura" en el sentido de que sobrepasa las preferencias individuales e influye en el desarrollo tecnológico (Castells, 2001).

Por otro lado, el peso de este tipo de usuarios también se nota directamente en el desarrollo puramente técnico del estándar. Como el propio W3C describe, algunos de los nuevos elementos semánticos de HTML5 están influenciados por la estructura de estas nuevas pla-

\footnotetext{
${ }^{10}$ Entendemos como usuario no técnico, aquel que no dispone de conocimientos de programación web. Es de cir, ausencia de conocimientos de lenguajes como HTML, CSS, JavaScript, PHP, Pearl, Python, etc.

${ }^{11}$ Un Mash-Up es una aplicación web, que se sirve de otras fuentes (a través de una API) para crear un servicio de agregación o de valor añadido. El sitio web http://digg.com/ es un ejemplo de Mash-Up.
} 
taformas. Es el caso del elemento article, que está diseñado en base a los posts de un blog (W3C, 2013) o del elemento aside, que recuerda en gran medida a la funcionalidad del blogroll $^{12}$. Y es que los blogs (Schafer, 2010) y otros medios sociales han influenciado sobremanera en el desarrollo del estándar. El uso que hacen millones de usuarios no técnicos de estas herramientas propicia la adecuación del estándar a su realidad de uso.

\section{Conclusiones}

En el presente artículo hemos explorado el proceso de creación y desarrollo de HTML5 desde una perspectiva tecnológica y social. El objetivo ha sido el de proporcionar visiones complementarias que ayuden a contextualizar el desarrollo de HTML5. Como hemos visto, se pueden encontrar elementos característicos del modelo SCOT y al mismo tiempo, algunos de los efectos no deseados de la "Tecnología Autónoma". En contraposición a esta dualidad, también hemos explorado el papel de los usuarios no técnicos en el proceso de creación del nuevo estándar. En especial, su influencia oculta en la conceptualización del nuevo estándar y en el paradigma de innovación que rige actualmente el desarrollo de los estándares web.

En nuestro trabajo sugerimos que los usuarios no técnicos han influido decisivamente a través de la adopción y el uso de medios sociales o "Social Media" en;

- La popularización y apropiación social de la World Wide Web.

- La vuelta a la estandarización y el abandono de software propietario a favor de HTML5.

- La primacía de contenidos multimedia en la World Wide Web.

Si bien esta influencia no se produce de manera directa (los usuarios no editan directamente la Web) y a pesar de que la cantidad y complejidad de las relaciones entre actores hace difícil definir una interdependencia clara entre factores. No obstante, creemos que el número creciente de usuarios y su preferencia por crear y compartir determinados tipos de contenidos son una de las grandes palancas de cambio que existen hoy en día en la Web. Su influencia es manifiesta para que desarrolladores, distribuidores de software, instituciones y demás agentes implicados apuesten por un desarrollo tecnológico que prime la eficiencia, la

\footnotetext{
${ }^{12}$ El blogroll es una lista de enlaces que se sitúa en uno de los laterales de un blog, a modo de lista de "blogs amigos".
} 
interoperabilidad, el acceso distribuido y la retro-compatibilidad, valores tecnológicos y sociales que han estado presentes desde los inicios de la World Wide Web

Por todo ello, creemos que el presente texto aporta una mayor comprensión del papel del usuario medio en el desarrollo tecnológico de la World Wide Web y de la expansión del estándar HTML. En este sentido, también pensamos que se abre un nuevo horizonte para el estudio social de estándares (Tabarés-Gutiérrez, 2015), ya que lo más destacable del surgimiento de HTML5 es el modo participativo e inclusivo bajo el cual se ha fraguado. 


\section{Referencias}

Alonso, I., \& GArcía, J. (2012). La contribución empresarial al software libre como actividad socialmente responsable. Teknokultura. Revista de Cultura Digital y Movimientos Sociales, 10(2013), 221-230. Extraído de:

$<$ http://teknokultura.net/index.php/tk/article/view/62>

Anderson, C. (2004). The Long Tail. Wired. Extraído de:

$<$ http://www.wired.com/wired/archive/12.10/tail.html> [22 de Marzo, 2014]

Anderson, C. (2007). La economía “Long Tail”. De los mercados de masas al triunfo de lo minoritario. Ediciones Urano.

Anderson, P. (2007). What is Web 2 . 0 ? Ideas, technologies and implications for education by. Extraído de:

$<$ http://www.jisc.ac.uk/media/documents/techwatch/tsw0701b.pdf $>$

Andersson, D. (2007). HTML5, XHTML2, and the Future of the Web. Digital Web Magazine. Extraído de:

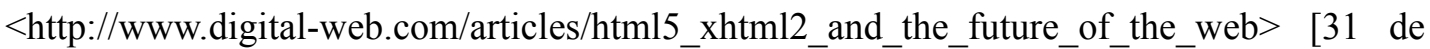
Diciembre, 2013]

Benkler, Y. (2006). The Wealth of Networks: How Social Production Transforms Markets and Freedom. New Haven and London: Yale University Press.

Berners-Lee, T. (2006). Reinventing HTML. Dig Research Blog. Extraído de: $<$ http://dig.csail.mit.edu/breadcrumbs/node/166> [20 de Abril, 2016]

BiJker, W. E. (1995). Sociohistorical Technology Studies. In S. Jasanoff, G. Markle, J. Petersen, \& T. Pinch (Eds.), Handbook of science and technology studies, revised edition. (pp. 229257). Thousand Oaks, CA: SAGE Publications.

Bijker, W. E., Hughes, T. P., \& Pinch, T. J. (1987). The social construction of technological systems: New directions in the sociology and history of technology. (W. E. Bijker, T. P. Hughes, \& T. J. Pinch, Eds.). Cambridge, MA: MIT Press.

Bijker, W. E., \& Pinch, T. J. (1984). The Social Construction of Facts and Artefacts : or How the Sociology of Science and the Sociology of Technology might Benefit Each Other. Social Studies of Science, 14(3), 399-441.

CAstells, M. (2001). La Galaxia Internet. Barcelona: Areté. 
Castro, E. (2007). HTML, XHTML y CSS. Anaya Multimedia.

Chau, M. (2009). HTML5: Its Evolution and Why It Matters. Itsc.org.sg, 9-20. Extraído de: $<$ http://www.itsc.org.sg/pdf/2011/Section2_Article1_021111.pdf>

Duggan, M., \& Smith, A. (2014). Three Technology Revolutions. Pew Research Internet Project. Extraído de:

$<$ http://www.pewinternet.org/three-technology-revolutions/> [22 de Marzo, 2014]

Echeverría, J. (2008). El manual de Oslo y la innovación social. Arbor, 184(732), 609-618. Extraído de:

$<$ http://arbor.revistas.csic.es/index.php/arbor/article/viewArticle/210>

ECHEVERRÍA, J. (2013). Evaluar las innovaciones y su difusión social. Isegoría, (48), 173-184. doi:10.3989/isegoria.2013.048.09

Ellul, J. (1964). The Technological Society. Vintage Books.

Estalella, A., Rocha, J., \& Lafuente, A. (2013). Laboratorios de procomún: experimentación, recursividad y activismo. Teknokultura, 10, 21-48. Extraído de:

$<$ http://teknokultura.net/index.php/tk/article/view/121/pdf $>$

Franganillo, J. (2010). Html5: el nuevo estándar básico de la Web. In Anuario Think EPI (Vol. 5, pp. 261-265). Extraído de:

$<$ http://www.academia.edu/download/30940232/html5.pdf $>$

González García, M., López Cerezo, J. A., \& Luján, J. L. (1996). Las concepciones de la tecnologia. In Ciencia, Tecnología y Sociedad. Una introducción al estudio social de la ciencia y la tecnología (pp. 1-16). Madrid: TECNOS.

GutiérRez, R. T. (2012). El inicio de la Web: historia y cronología del hipertexto hasta HTML 4.0 (1990-99). ArtefaCToS, 5(1), 57-82. Extraído de:

$<$ http://rca.usal.es/ revistas_trabajo/index.php/artefactos/article/view/12423>

Hackett, E. J., Amsterdamska, O., Lynch, M. E., \& Wajcman, J. (2007). The Handbook of Science and Technology Studies. (E. J. Hackett, O. Amsterdamska, M. E. Lynch, \& J. Wajcman, Eds.) (3rd ed.). Cambridge, MA: The MIT Press.

Helmer, O., Brown, B., \& Gordon, T. (1966). Social Technology. New York: Basic Books.

Hickson, I., \& Hyatt, D. (2008). HTML 5 A vocabulary and associated APIs for HTML and XHTML (W3C Working Draft 22 January 2008). W3C Working Draft. Extraído de: $<$ http://www.w3.org/TR/2008/WD-html5-20080122/> 
Hughes, T.P. (1987). The evolution of large technological systems. In W. E. Bijker, T. P. Hughes, \& T. J. Pinch (Eds.), The Social Construction of Technological Systems (pp. 5182). Cambridge, MA: MIT Press.

InNerarity, D., \& GurrutXaga, A. (2009). ¿Cómo es una sociedad innovadora? Zamudio: Innobasque.

JoBs, S. (2010). Thoughts on Flash. Apple Press. Extraído de:

$<$ http://www.apple.com/hotnews/thoughts-on-flash/>

KeITH, J. (2010). HTML5 for web designers. Nueva York: A Book Apart.

KLemperer, P. (2006). Network Effects and Switching Costs: Two Short Essays for the New Palgrave. SSRN Electronic Journal.

doi:10.2139/ssrn.907502

Lafuente, A. (2007). Los cuatro entornos del procomún. Cuadernos de Crítica de La Cultura, (77-78), 15-22. Extraído de:

$<$ http://digital.csic.es/handle/10261/2746>

Lafuente, A., \& Jiménez, A. C. (2010). Comunidades de afectados, procomún y don expandido. Fractal, 57, 10-42.

Laningham, S. (2006, August 22). developerWorks Interviews: Tim Berners-Lee. Ibm developerWorks. Extraído de:

$<$ http://www.ibm.com/developerworks/podcast/dwi/cm-int082206txt.html $>$ [15 de Marzo, 2014]

Latour, B. (1992). Ciencia en acción. Barcelona: Labor.

Le Hegaret, P. and I. J. (2009). Frequently Asked Questions (FAQ) about the future of XHTML. W3C FAQ. Extraído de:

$<$ http://www.w3.org/2009/06/xhtml-faq.html $>$ [22 de Abril, 2016]

Liebowitz, S. J., \& Margolis, S. E. (1994). Network Externality: An Uncommon Tragedy. Journal of Economic Perspectives, 8(2). Extraído de:

$<$ http://www.utdallas.edu/ liebowit/jep.html $>$

Linder, B. (2013). Cisco sets H.264 free(ish) with royalty-free video codec. Liliputing. Extraído de:

$<$ http://liliputing.com/2013/10/cisco-sets-h-264-freeish-with-royalty-free-video-codec.html> [22 de Marzo, 2014] 
López Cerezo, J. a., \& GonzÁlez, M. I. (2013). Encrucijadas sociales de la innovación. Isegoría, (48), 11-24.

doi:10.3989/isegoria.2013.048.01

Lundvall, B.-A. (1992). National Systems of Innovation: Towards a Theory of Innovation and Interactive Learning. London: Pinter Publ.

O'Mara, M.P. (2012). New Features In HTML5. University of Wisconsin-Platteville (Department of Computer Science).

O'Reilly, T. (2005). What Is Web 2.0? Design Patterns and Business Models for the Next Generation of Software. O'Reilly. Extraído de:

$<$ http://oreilly.com/web2/archive/what-is-web-20.html> [22 de Marzo, 2014]

OECD. (2005). Manual de Oslo: Guía para la recogida e interpretación de datos sobre innovación. Tragsa. Extraído de:

$<$ http://www.uis.unesco.org/Library/Documents/OECDOsloManual05_spa.pdf>

Pilgrim, M. (2010). HTML5: Up and Running. O’Reilly Media.

Quintanilla, M.A. (1998). Técnica y cultura. Teorema, XVII/3. Extraído de:

$<$ http://www.oei.es/salactsi/teorema03.htm>

Quintanilla, M.A. (1989). Tecnología: Un enfoque filosófico. Madrid: FUNDESCO.

Rogers, E.M. (1962). Diffusion of Innovations. Free Press.

SchAFER, S.M. (2010). HTML, XHTML y CSS. Madrid: Anaya Multimedia.

Schonfeld, E. (2010). Google, Mozilla, And Opera Take On H.264 With The WebM Project, A New Royalty-Free Video Codec. TechCrunch. Extraído de:

$<$ http://techcrunch.com/2010/05/19/webm-google-h-264/> [22 de Marzo, 2014]

Shapiro, C., \& Varian, H. R. (1999). Information Rules. Harvard Business School Press.

Tabarés-Gutiérrez, R. (2015, July 29). La Belleza del Código: Influencia de la Web 2.0, los medios sociales y los contenidos multimedia en el desarrollo de HTML5. Universidad de Salamanca. Extraído de:

$<$ http://dsp.tecnalia.com/handle/11556/190>

The Mozzilla Foundation \& Opera Software. (2004). Position Paper for the W3C Workshop on Web Applications and Compound Documents. W3C Workshop on Web Applications and Compound Documents. Extraído de:

$<$ http://www.w3.org/2004/04/webapps-cdf-ws/papers/opera.html> [23 de Diciembre, 2013] 
Tucker, J.V., Wang, V., \& Haines, K. (2012). Phatic technologies in modern society. Technology in Society, 34(1), 84-93. doi:10.1016/j.techsoc.2012.01.001

Von HipPel, E. (1998). The Sources of Innovation. Oxford: Oxford University Press.

Von Hippel, E. (2005). Democratizing Innovation. Cambridge, MA: The MIT Press. Extraído de: $<$ http://web.mit.edu/evhippel/www/democ1.htm>

W3C. (2011). HTML5. W3C Recommendation. Extraído de: $<$ http://www.w3.org/TR/html5/> [23 de Abril, 2016]

W3C. (2013). Differences from HTML4 (W3C Working Draft 28 May 2013). W3C Working Group Note. Extraído de: $<$ http://www.w3.org/TR/html5-diff/> [2 de Enero, 2014]

W3C. (2014). Open Web Platform Milestone Achieved with HTML5 Recommendation. Extraído de: $<$ http://www.w3.org/2014/10/html5-rec.html.en> [18 de Enero, 2015]

Wang, V., Tucker, J. V., \& Rihll, T. E. (2011). On phatic technologies for creating and maintaining human relationships. Technology in Society, 33(1-2), 44-51. doi:10.1016/j.techsoc.2011.03.017

Williams, R., \& Edge, D. (1996). The social shaping of technology. Research Policy, 25(6), 865-899. doi:10.1016/0048-7333(96)00885-2

WINNER, L. (1979). Tecnología Autónoma. Barcelona: Gustavo Gilli.

Winner, L. (1980). Do Artifacts Have Politics? Daedalus, 109(1), 121-136. Extraído de: $<$ https://blog.itu.dk/I-II-E2013/files/2013/11/winner-1-do-artifacts-have-politics.pdf>

WinNER, L. (1993). Upon opening the black box and finding empty: Social Constructivism and the Philosophy of Technology. Science, Technology \& Human Values, 18(3), 362-378. 